\title{
Administration of erythropoietin exerts protective effects against glucocorticoid-induced osteonecrosis of the femoral head in rats
}

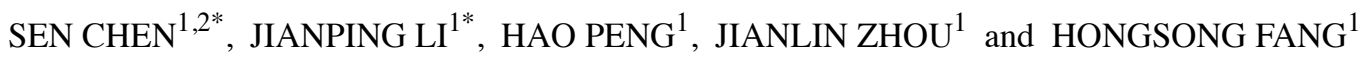 \\ ${ }^{1}$ Department of Orthopedics, Renmin Hospital of Wuhan University, Wuhan, Hubei 430060; \\ ${ }^{2}$ Dongfeng General Hospital, Hubei University of Medicine, Shiyan, Hubei 442008, P.R. China
}

Received September 21, 2013; Accepted January 23, 2014

DOI: $10.3892 /$ ijmm.2014.1644

\begin{abstract}
Accumulating evidence has indicated that erythropoietin (EPO) plays a role in anti-apoptosis and tissue protection in a number of human diseases. The present study was implemented to evaluate these anti-apoptotic and tissueprotective effects in glucocorticoid-induced osteonecrosis in rats. Osteonecrosis was induced by low-dose lipopolysaccharide and subsequent high-dose methylprednisolone pulse. Rats in the preventive group were treated with $500 \mathrm{U} / \mathrm{kg} /$ day recombinant human EPO (rhuEPO) for 1 week. Hematological and histomorphometric methods were then used to determine the effects of the administration of rhuEPO. An analysis of trabecular bone architecture was performed to evaluate bone mass change in the osteonecrosis zone. Terminal deoxynucleotidyl transferase-mediated dUTP nick end-labeling (TUNEL) assay was performed to determine the apoptotic index of osteoblasts and osteocytes. Immunoblot analysis was performed to assess the expression of caspase- 3 and vascular endothelial growth factor (VEGF) in the femoral head. Treatment with rhuEPO greatly improved the histological performance. Additionally, the incidence of osteonecrosis markedly decreased in the rats in the rhuEPO-treated group (22.2\%) compared with the control group (66.7\%). Furthermore, the expression of caspase-3 markedly decreased in the rhuEPO-treated group. Consistently, the apoptosis of osteoblasts and osteocytes, as determined by TUNEL assays, was inhibited following the administration of rhuEPO. By contrast, the expression of VEGF increased in the osteonecrosis zone in the rats treated with rhuEPO. The results from the present study demonstrate that EPO exerts prominent protective effects against glucocorticoid-induced osteonecrosis
\end{abstract}

Correspondence to: Dr Hao Peng, Department of Orthopedics, Renmin Hospital of Wuhan University, 238 Jiefang Road, Wuhan, Hubei 430060, P.R. China

E-mail: penghao5868@163.com

*Contributed equally

Key words: erythropoietin, glucocorticoid, osteonecrosis, rat of the femoral head in rats by inhibiting the apoptosis of osteoblasts and osteocytes and increasing the expression of VEGF.

\section{Introduction}

Osteonecrosis $(\mathrm{ON})$ is a pathological process that predominantly impairs the femoral head and gradually progresses to the fracture of the subchondral bone, the collapse of the femoral head surface and the destruction of the hip joint. Although the etiology of $\mathrm{ON}$ has been attributed to a number of factors, glucocorticoid (GC) administration is the predisposing causative factor most commonly associated with the development of ON. Compromised vascularity and bone ischemia represent the traditional etiological background of ON. It was then realized that osteoblast and osteocyte apoptosis, but not compromised vascularity, is the primary etiology of GC-induced ON (1-12). In addition, the expression of vascular endothelial growth factor (VEGF), a key regulator that couples angiogenesis, bone formation and repair, reduces accompanied by the increase of osteoblasts and osteocytes apoptosis (13). Also, the compromised expression of VEGF has been found to impair the bone regeneration in the necrotic zones of the femoral head (14). On the basis of the above considerations, suppression of the apoptosis of the osteoblast and osteocytes and stimulation of the expression of VEGF could be two effective attempts in preventing the GC-induced ON of the femoral head.

Erythropoietin (EPO) is a pleiotropic cytokine originally identified for its role in erythropoiesis (15). Accumulating evidence has indicated that erythropoietin exerts anti-apoptotic and tissue-protective effects in a variety of human diseases, such as myocardial infraction (16-18), diabetes mellitus (19), spinal cord injury (20-22), ischemia-reperfusion (I/R)-induced kidney injury (23) and acute lung injury $(24,25)$. Galeano et al reported that recombinant human EPO (rhuEPO) may be an effective therapeutic approach for improving clinical outcomes by enhancing the wound content of vascular endothelial growth factor (VEGF) following thermal injury (26). Rezaeian et al also demonstrated that the pharmacological manipulation of ischemic musculocutaneous tissue with 3 repetitive doses of EPO (500 IU/kg) upregulated inducible nitric oxide synthase (iNOS) and VEGF expression, and reduced apoptotic cell death and inflammation in the absence of any hematopoietic effect (27). Additionally, Holstein et al found that treatment 
with EPO upregulated the expression of VEGF during the early phase of bone defect healing, as shown by immunoblot and immunohistochemistry analyses (28). These data suggest that EPO exerts tissue protective effects through a VEGFrelated pathway.

Therefore, we hypothesized that the administration of EPO can protect the femoral head from GC-induced ON by inhibiting apoptosis and increasing the expression of VEGF. We investigated this hypothesis using rats and a variety of methods, including histological staining and protein biochemistry. Indeed, we found that the administration of EPO markedly reduced the incidence of GC-induced $\mathrm{ON}$ in rats. Moreover, EPO suppressed the apoptosis of osteoblasts and osteocytes and increased the expression of VEGF.

\section{Materials and methods}

Animals. All experimental procedures adhered to the recommendations of the Experimental Animal Center of Wuhan University, Wuhan, China and the US Department of Health Guide for the Care and Use of Laboratory Animals, and were approved by the Ethics Committee of Wuhan University. A total of 54 male Wistar rats (10 weeks old) were obtained from the Hubei Provincial Center for Disease Control and Prevention, Wuhan, China. The rats were housed in a temperature- and humidity-controlled environment with unlimited access to food and water and a 12-h light/dark cycle.

Experimental protocols. A total of 54 rats were divided equally into 2 groups: the control and EPO group. A rat model of ON was created by a sequential drug administration. The animals were administered $2 \mathrm{mg} / \mathrm{kg}$ lipopolysaccharide (LPS, from Escherichia coli 055: B5; Sigma, St. Louis, MO, USA) intravenously on days 0 and 1 . On days 2, 3 and 4, the animals were administered $20 \mathrm{mg} / \mathrm{kg}$ methylprednisolone (MPS; Pfizer Pharmaceutical, Puurs, Belgium) intramuscularly. The animals in the EPO group were administered $500 \mathrm{U} / \mathrm{kg}$ rhuEPO (Shenyang Sunshine Pharmaceutical Co. Ltd., Shenyang, China) intramuscularly daily from day 0 for 7 days. The final day of administration was regarded as experimental week 0 . The rats in the control group were not administered EPO. Nine rats in each group were sacrificed (6 for histological analysis and 3 for molecular biological analysis) on weeks 0, 2 and 4 .

Blood biochemistry. Blood was collected from the inferior vena cava at the time of sacrifice partially for regular testing and the remaining blood was centrifuged immediately. The supernatant was stored as platelet-rich plasma at $-80^{\circ} \mathrm{C}$. The triglyceride concentrations in the plasma were measured by using Triglyceride E-test kit (Nanjing Jiancheng Bioengineering Institute, Nanjing, China) according to the manufacturer's instructions. The total cholesterol levels in the plasma were measured using the Cholesterol E-test kit (Nanjing Jiancheng Bioengineering Institute) according to the manufacturer's instructions.

Histopathological analysis. The proximal femurs were harvested and fixed with $10 \%$ formalin- $0.1 \mathrm{M}$ phosphate buffer, $\mathrm{pH}$ 7.4. After fixing in formalin, the bone samples were decalcified in $10 \%$ EDTA for 2 months. The decalcified bones were then embedded in paraffin and sectioned at $5 \mu \mathrm{m}$ for histopathological analysis and immunohistochemistry. The sections were processed for routine haematoxylin and eosin staining to evaluate the ON lesions using well established criteria (29-32). The diagnosis of $\mathrm{ON}$ was performed in a blinded manner by 3 of the authors on the basis of the diffuse presence of empty lacunae or pyknotic nuclei of osteocytes in the bone trabeculae, accompanied by surrounding bone marrow cell necrosis. Rats that had at least $1 \mathrm{ON}$ lesion in the areas examined were considered as $\mathrm{ON}^{+}$, while those with no $\mathrm{ON}$ lesions were considered as $\mathrm{ON}^{-}$. The incidence of $\mathrm{ON}$ was defined as the numbers of $\mathrm{ON}^{+}$rats divided by the numbers of total rats in each group.

Trabecular bone architecture. Frontal sections (4- $\mu \mathrm{m}$-thick) of each femoral head were obtained and stained with Masson's trichrome to highlight the microstructure. The trabecular tissue area $\left(\mathrm{T} . \mathrm{Ar}, \mathrm{mm}^{2}\right)$, the trabecular bone area (B.Ar, $\mathrm{mm}^{2}$ ) and trabecular perimeter (B.Pm, $\mathrm{mm}$ ) were quantified for each section in the central region of the proximal epiphysis; the bone volume fraction (\%) was calculated as the trabecular bone area (BV) divided by the trabecular tissue area (TV) (Fig. 1). Other architectural properties, including trabecular thickness (Tb.Th, mm), trabecular number (Tb.N, mm-1) and trabecular separation (Tb.Sp, $\mathrm{mm}$ ) were calculated according to a parallel plate model $(33,34)$.

TUNEL assays. Apoptotic osteoblasts and osteocytes were detected using the terminal deoxynucleotidyl transferasemediated dUTP nick end-labeling (TUNEL) assay, with an In Situ Cell Death Detection Kit (Roche Diagnostics, Mannheim, Germany), according to the manufacturer's instructions. Briefly, following routine deparaffinization and treatment with $\mathrm{H}_{2} \mathrm{O}_{2}(3 \%)$, the sections were digested with proteinase $\mathrm{K}(20 \mu \mathrm{g} / \mathrm{ml}, \mathrm{pH} 7.4,12 \mathrm{~min})$ at $25^{\circ} \mathrm{C}$ and incubated with the reaction mixture $(1: 40,60 \mathrm{~min})$ at $37^{\circ} \mathrm{C}$. Incorporated fluorescein was detected with horseradish peroxidase following incubation for $30 \mathrm{~min}$ at $37^{\circ} \mathrm{C}$ and were subsequently dyed with 3,3'-diaminobenzidine (DAB). Brown nuclei were assessed as positive apoptotic cells. The apoptotic index (AI) was evaluated for 1 section of 5 randomly selected high-power fields.

Immunoblot analysis. Femoral heads dissected from the proximal one-third of the femur neck were powdered in liquid nitrogen by hand milling, followed by homogenization on ice-cold radioimmunoprecipitation (RIPA, Beyotime Institute of Biotechnology, Beijing, China) buffer containing phenylmethylsulfonyl fluoride (PMSF, Beyotime Institute of Biotechnology) and a cocktail of protease inhibitors (Complete, EDTA-free; Roche Diagnostics). Following sonication, the samples were centrifuged twice at $14000 \mathrm{rpm}$ at $4^{\circ} \mathrm{C}$ for $10 \mathrm{~min}$ to remove cell debris, nuclei and large particulates. The supernatant containing the cytosolic protein fraction was then collected. A quarter volume of $5 \mathrm{X}$ loading buffer was added and boiled at $95^{\circ} \mathrm{C}$ for $5 \mathrm{~min}$ then stored at $-20^{\circ} \mathrm{C}$ until electrophoresis. Proteins were separated by $10 \%$ sodium dodecyl sulfate polyacrylamide gel electrophoresis and transferred onto polyvinylidene difluoride membranes (Millipore Corp., Bedford, MA, USA). After being blocked with $2 \%$ bovine serum albumin (Roche Diagnostics), the membranes were 

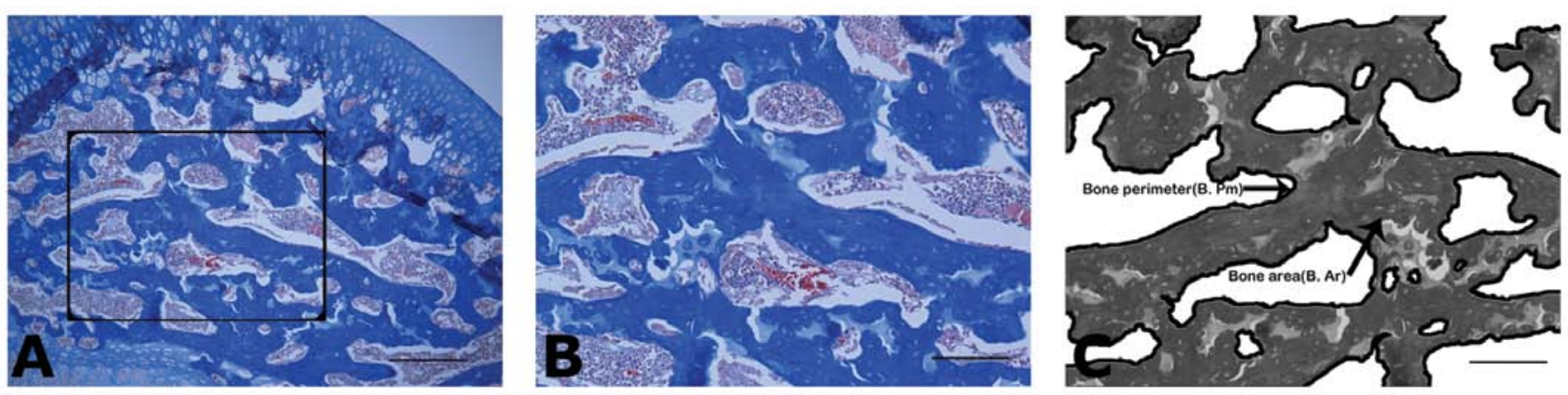

Figure 1. Trabecular bone microstructure in the femoral head. (A) The central region of the proximal epiphysis was selected for analysis. (B) The selected area was enlarged. (C) Digital image used for the quantification of trabecular architecture. Tissue area, trabecular bone area and trabecular bone perimeter were quantified and used to calculate trabecular bone architectural characteristics according to a parallel plate model. BV, bone volume; TV, tissue volume. Bone volume fraction $(\mathrm{BV} / \mathrm{TV})=\mathrm{B} . \mathrm{Ar} / \mathrm{T} . \mathrm{Ar}$; trabecular thickness $(\mathrm{Tb} . \mathrm{Th})=[2 /(\mathrm{B} . \mathrm{Pm} / \mathrm{B} . \mathrm{Ar}) \mathrm{x} 1,000]$; trabecular number $(\mathrm{Tb} . \mathrm{N})=(\mathrm{BV} / \mathrm{TV}) / \mathrm{Tb} . \mathrm{Th}$; trabecular separation $(\mathrm{Tb} . \mathrm{Sp})=(1 / \mathrm{Tb} . \mathrm{N})-\mathrm{Tb} . T h$. Scale bar, (A) $400 \mu \mathrm{m}$; (B and C) $200 \mu \mathrm{m}$.

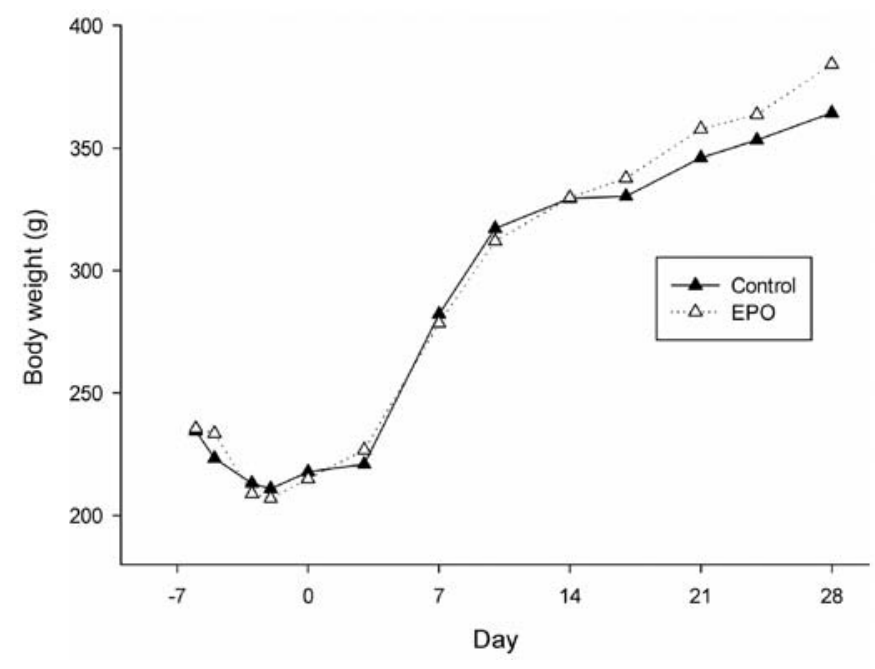

Figure 2. Body weight over time of rats in the control and erythropoietin (EPO) groups.

incubated at $4^{\circ} \mathrm{C}$ overnight with rabbit anti-caspase- 3 or antiVEGF antibody (1:500, Santa Cruz Biotechnology, Santa Cruz, CA, USA) or rabbit anti-GAPDH antibody (1:500, Santa Cruz Biotechnology) as primary antibodies, followed by incubation with peroxidase-conjugated secondary antibodies (1:2000, Jackson Laboratories, West Grove, PA, USA) at $25^{\circ} \mathrm{C}$ for $1 \mathrm{~h}$. The proteins on the membranes were visualized using an ECL plus detection kit (Amersham Pharmacia Biotech, Buckinghamshire, UK), exposed to Kodak X-ray film and processed using a scanner. The optical density of the bands was analyzed using Image-Pro Plus 6.0 software. The expression of caspase-3 and VEGF was then normalized to GAPDH.

Statistical analysis. Data are presented as the means \pm standard error of the mean (SEM). Statistical analysis was performed using SPSS 13.0 software (SPSS Inc., Chicago, IL, USA). One-way analysis of variance (ANOVA) with Turkey's post hoc test was used to examine differences between groups. Statistical differences were considered significant when the P-value was $<0.05$.

\section{Results}

EPO administration does not affect body weight, red blood cell (RBC) count, hemoglobin and hematocrit levels in rats. No accidental deaths took place during the experiment. The body weight of the animals in both groups decreased slightly during the 1st week (week, -1 ), and thereafter increased from weeks 0 to 4 . There were no significant differences between the body weight of the animals in the 2 groups during the whole experimental period (Fig. 2). Blood analyses did not reveal a significantly higher RBC count in the rhuEPO-treated animals when compared with the controls. Additionally, the levels of hemoglobin and hematocrit displayed a similar trend (Fig. 3), as the statistical analysis revealed no significant differences between the groups. These data indicate that the appropriate dose of EPO does not affect body weight and blood components in rats.

EPO administration decreases the plasma concentration of triglycerides and total cholesterol in rats. We then examined the concentrations of triglycerides and total cholesterol in plsma. As shown in Fig. 4, the plasma levels of triglycerides in the EPO group were lower than those in the control group. Notably, a significant difference was observed on week 2 $(\mathrm{P}<0.05)$. The level of total cholesterol in the plasma in the EPO group was also significantly lower than that in the control group on week 2 . These results suggest that the appropriate dosage of EPO reduces the plasma concentration of triglycerides and total cholesterol during the early stages of ON.

EPO administration improves histological performance and reduces the incidence of $O N$ in rats. The presence of diffuse and empty lacunae or pyknotic nuclei of osteocytes in the bone trabeculae, accompanied by surrounding bone marrow cell necrosis, is defined as ON. To assess the ON lesions in the control and EPO groups, we performed haematoxylin and eosin staining to determine the histological characteristics (Fig. 5). On week 0 , the necrotic bone trabeculae showed pyknotic nuclei of osteocytes and empty lacunae accompanied by the hemorrhage and necrosis of bone marrow (Fig. 5A and B). On week 2, the necrotic bone trabeculae also showed pyknotic nuclei and empty lacunae, while the numbers of empty lacunae markedly 

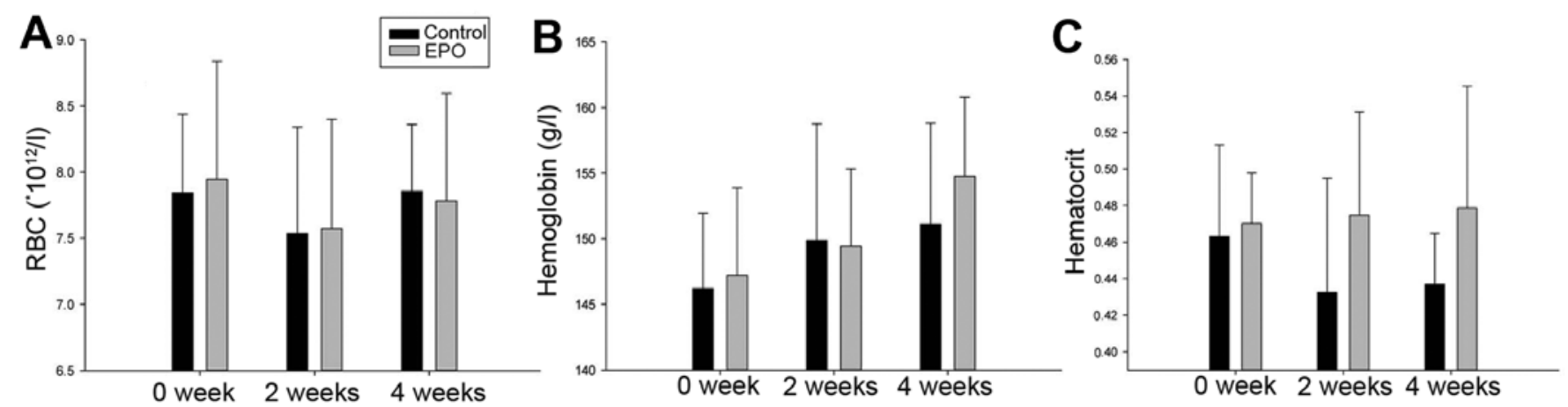

Figure 3. Blood tests over time in the control and erythropoietin (EPO) groups. (A) Red blood cell (RBC) count, (B) hemoglobin, (C) hematocrit. Results are presented as the means $\pm \mathrm{SD}$.
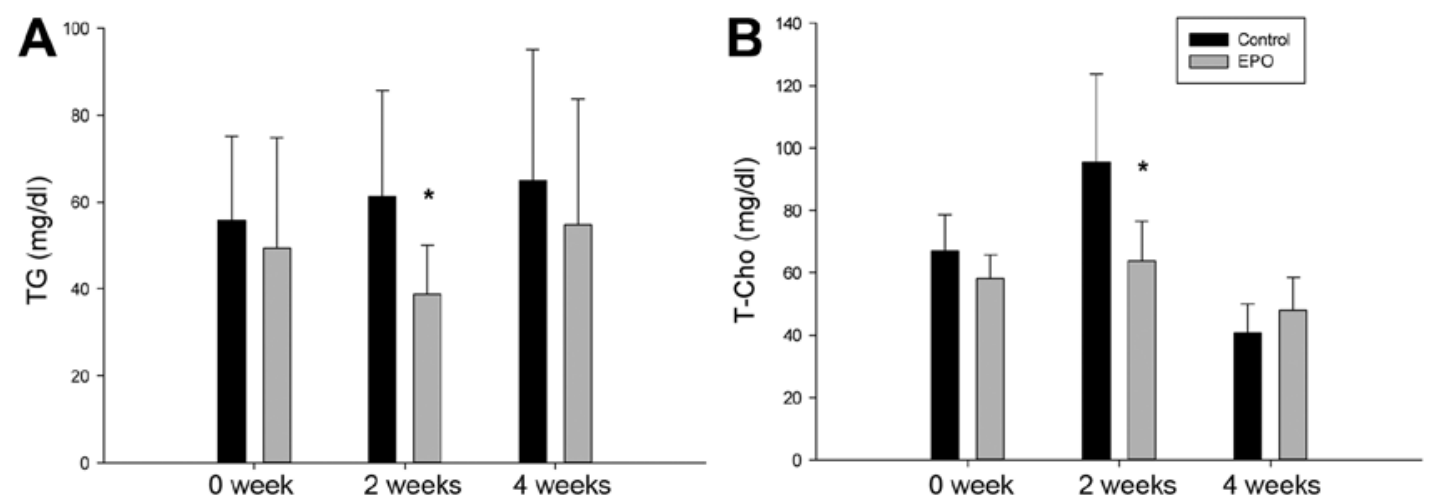

Figure 4. Blood biochemistry tests over time in the control and erythropoietin (EPO) groups. (A) Plasma levels of triglycerides (TG). (B) Level of total cholesterol (T-Cho) in the plasma. Results are presented as the means $\pm \mathrm{SD}$. ${ }^{*} \mathrm{P}<0.05$, compared to the control.
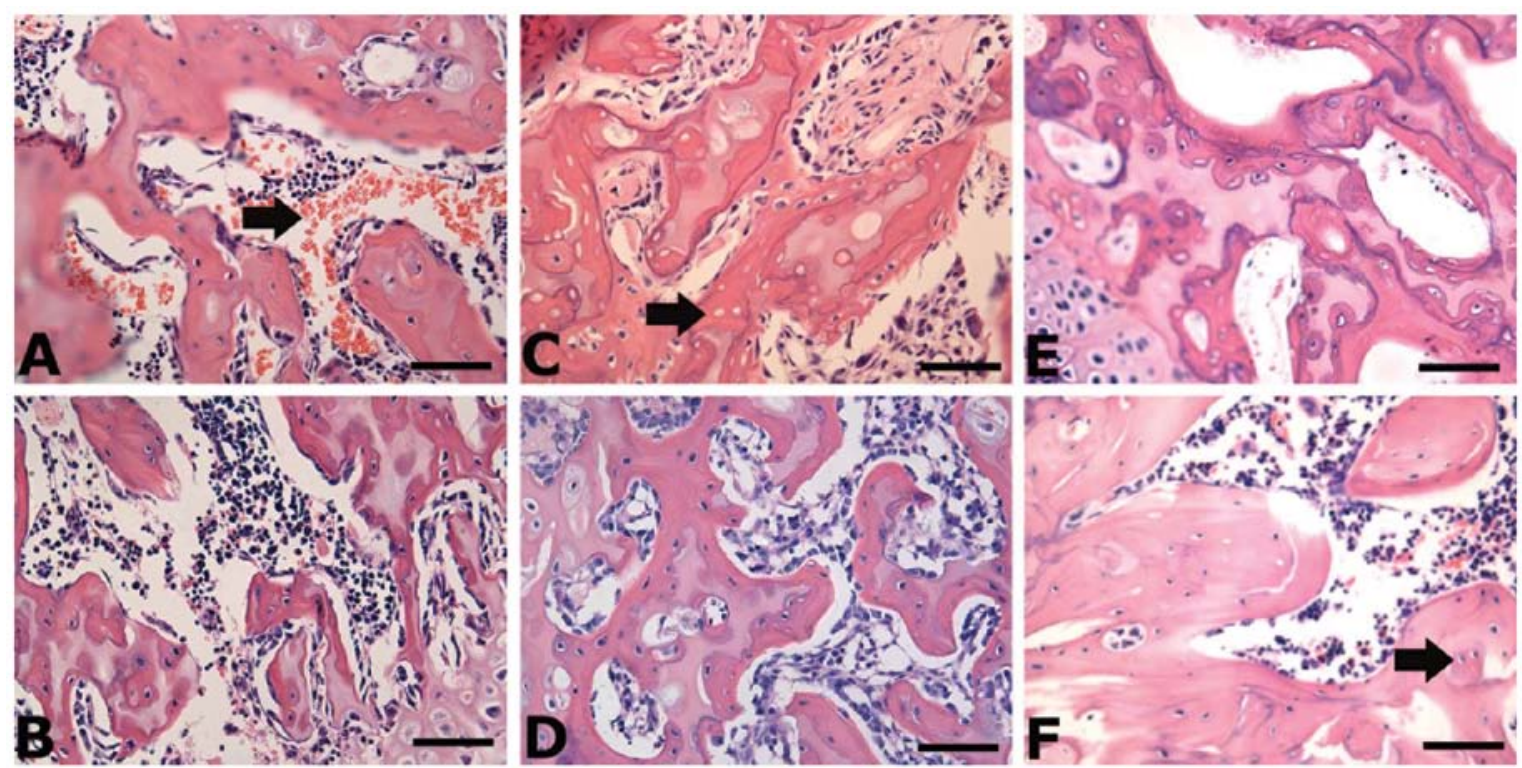

Figure 5. Histological changes of trabecular bone of femoral head. (A, C and E) Histological performance in the control group over time; (B, D and F) histological performance in the EPO group. Histological performance in the EPO group was much better than that in the control group. (A and B) On week 0, the necrotic bone trabeculae showed pyknotic nuclei of osteocytes and empty lacunae accompanied with the hemorrhage and necrosis of bone marrow. (C and D) On week 2, the necrotic bone trabeculae also showed pyknotic nuclei and empty lacunae, while the numbers of empty lacunae increased significantly. (E and F) On week 4 , the necrotic bone trabeculae again showed much more empty lacunae and the appearance of scar tissue in the medullary space. The arrow in (A) indicates the hemorrhage and necrosis of bone marrow. The arrows in (C and F) indicate empty lacunae and pyknotic nuclei of osteocytes. Scale bar, $100 \mu \mathrm{m}(\mathrm{A}-\mathrm{F})$.

increased (Fig. 5C and D). Additionally, the fibrous tissue was found to accumulate in the medullary space (Fig. 5C and D).
On week 4 , the necrotic bone trabeculae showed further empty lacunae and the presence of scar tissue in the medullary space 

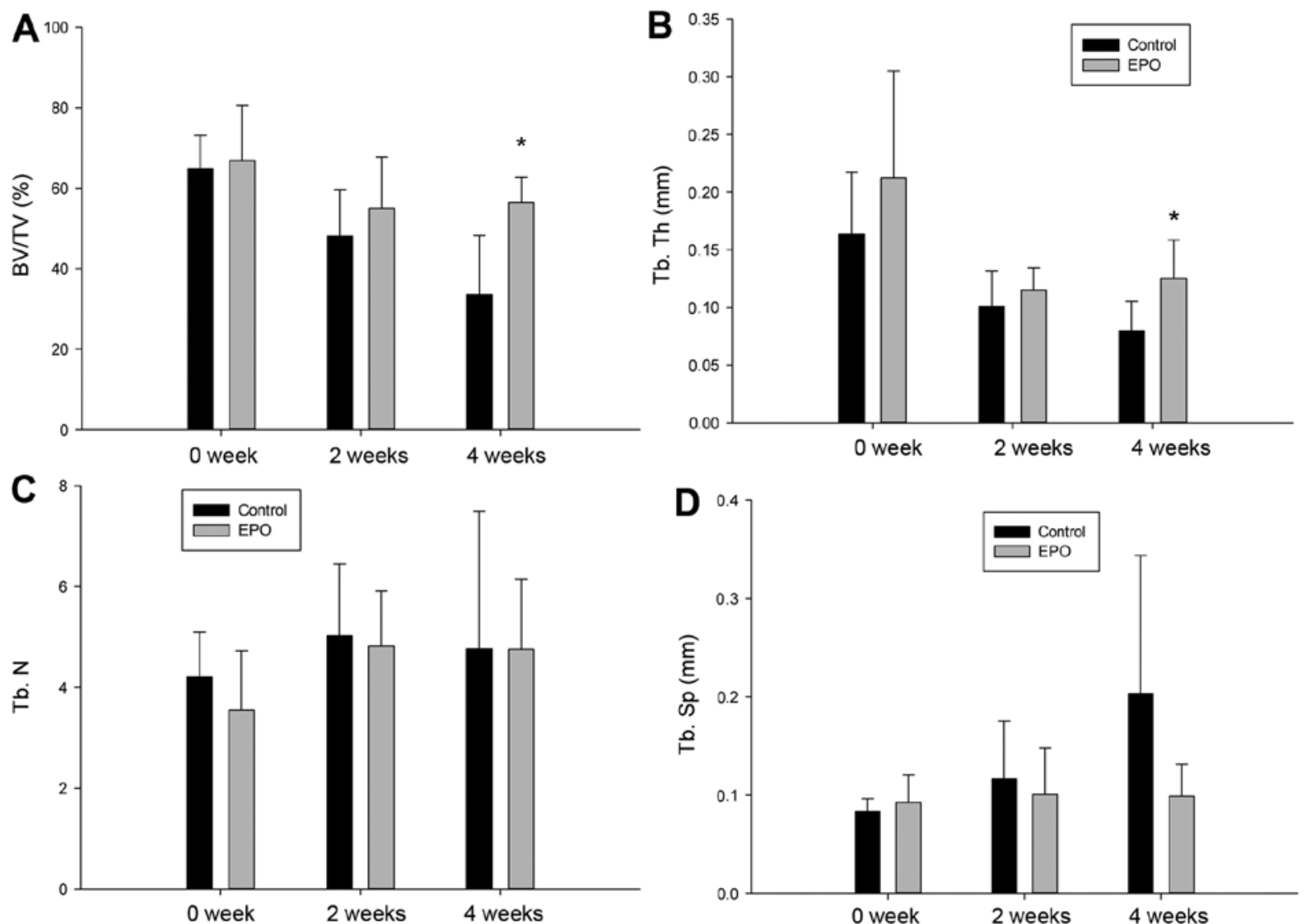

Figure 6. Trabecular bone microstructural properties. (A) Bone volume fraction (BV/TV) was significantly higher in the erythropoietin (EPO) group compared with the control group on week 4 ( $\mathrm{P}=0.006$ ), but not at other time points. (B) Bone trabecular thickness (Tb.Th) was significantly higher in the EPO group compared with the control group on week 4 ("P=0.023). (C) There was no significant difference in trabecular number (Tb.N) between the 2 groups at the 3 time points. (D) There was no significant difference in trabecular separation (Tb.Sp) between the 2 groups at the 3 time points.

Table I. Incidence of osteonecrosis (\%).

\begin{tabular}{lcccc}
\hline Group & Week 0 & Week 2 & Week 4 & Total \\
\hline Control & $33.3(2)$ & $66.7(4)$ & $100.0(6)$ & 66.7 \\
Erythropoietin & $16.7(1)$ & $33.3(2)$ & $16.7(1)$ & 22.2 \\
$\chi^{2}$ test & 0.4444 & 1.3333 & 8.5714 & 7.2000 \\
P-value & 0.5050 & 0.2482 & 0.0034 & 0.0073 \\
\hline
\end{tabular}

(Fig. 5E and F). Based on the histopathological characteristics, we determined the incidence of $\mathrm{ON}$ as presented in Table I. In the control group, the incidence of $\mathrm{ON}$ increased with time, whereas the administration of EPO prevented the occurrence of ON. The total incidence of ON markedly decreased in the EPO group compared with the control group (22.2 vs. $66.7 \%$ ) (Table I), suggesting that the appropriate dose of rhuEPO can greatly reduce the incidence of steroid-associated $\mathrm{ON}$ in rats.

Trabecular bone volume fraction and trabecular thickness increases upon EPO administration. To assess the microstructural architecture of the trabecular bone, we introduced 4 indicators, including bone volume fraction, trabecular thickness, trabecular number and trabecular separation, as described in Materials and methods. No significant difference was observed as regards the 4 indicators between the 2 groups on weeks 0 and 2 . Of note, the trabecular bone volume fraction and trabecular thickness in the EPO group increased with statistical significance on week $4(\mathrm{P}=0.006$ and $\mathrm{P}=0.023$; Fig. 6A and B). However, there were no significant differences observed in trabecular number and trabecular separation between the 2 groups (Fig. 6C and D).

EPO administration inhibits apoptosis in the ON zone. To determine whether the administration of EPO affects apoptosis in the trabecular bone of the femoral head, we conducted TUNEL assays and found that positively stained cells were detected in the ON zones in both groups, although the number of TUNEL-positive cells varied at different time points (Fig. 7A-F). Additionally, treatment with EPO resulted in less apoptotic cells/high-power field, which was further confirmed by quantitative analysis showing a significantly lower apoptotic index in the EPO group compared with the control group at all the 3 time points $(\mathrm{P}<0.05$; Fig. $7 \mathrm{G})$. These data indicate that the administration of EPO protects the trabecular bone cells from apoptosis.

EPO administration reduces caspase-3 and increases VEGF levels. To further consolidate our previous observations, we performed immunoblot analysis to assess the expressions of caspase-3, an indicator of apoptosis, as well as the levels of VEGF. Indeed, the expression of caspase-3 within the 

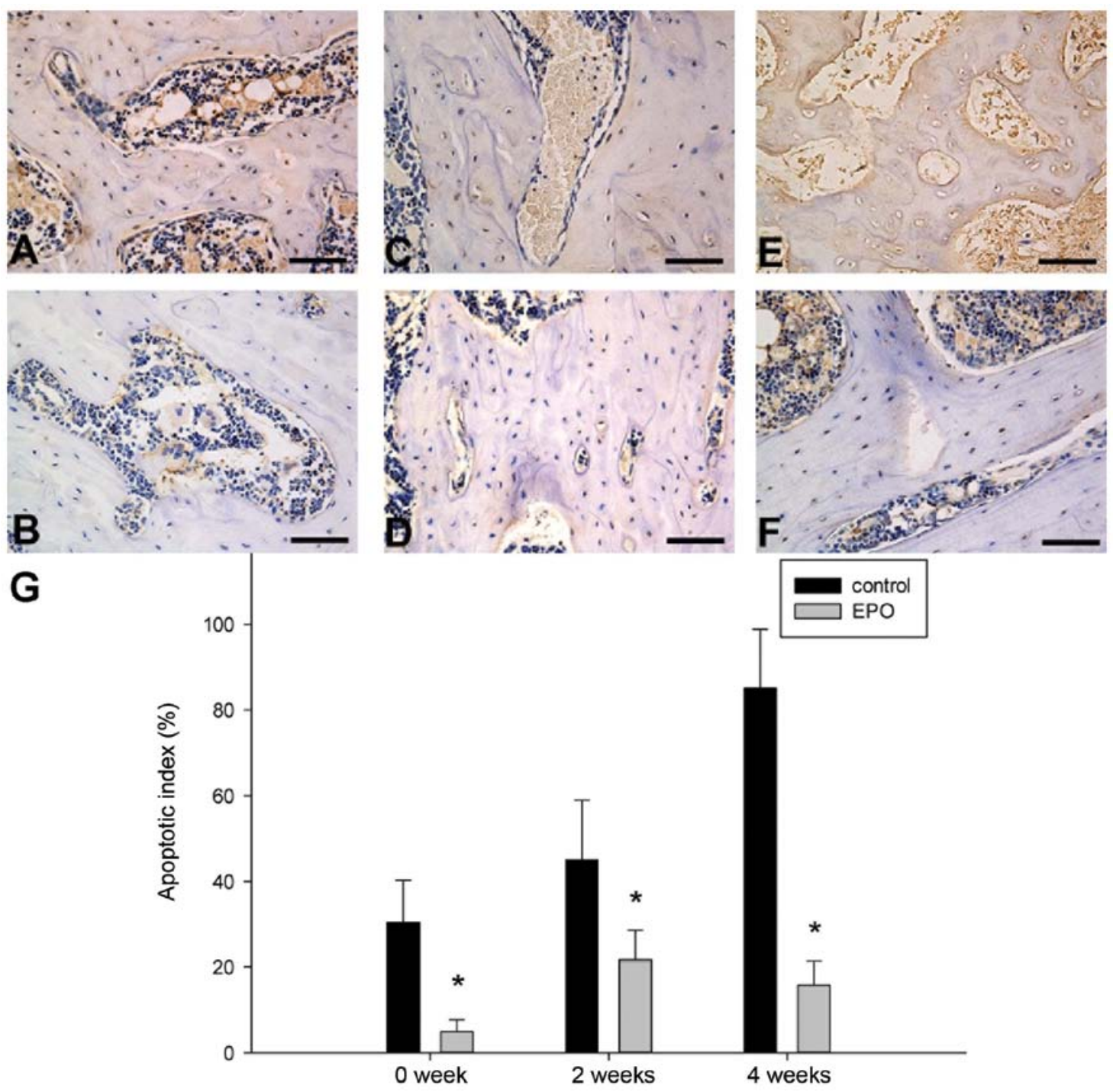

Figure 7. Photomicrograph of TUNEL staining showing evidence of apoptotic cells in the necrotic zone. (A, C and E) show the TUNEL-positive cells in the control group over time. (B, D and F) show the TUNEL-positive cells in the erythropoietin (EPO) group over time. Scale bar, $100 \mu \mathrm{m}$ (A-F). (G) Quantitative analysis of TUNEL-positive staining in (A-F). Data are presented as the means \pm SD. ${ }^{*} \mathrm{P}<0.05$ vs. controls.

femoral head markedly decreased in response to EPO treatment (Fig. 8A), while the difference was significant on week 0 and week $2(\mathrm{P}<0.05)$. On the contrary, the expression level of VEGF markedly increased following treatment with EPO on weeks 2 and 4 (Fig. 8B). These data demonstrate that EPO exerts tissue-protective effects against $\mathrm{ON}$ through 2 mechanisms, the inhibition of apoptosis and the enhancement of VEGF expression.

\section{Discussion}

EPO has been found to initiate tissue-protective effects, including the prevention of apoptotic cell death (35) and the induction of angiogenesis and tissue regeneration (36), in addition to its regulatory function in erythropoiesis (37). These effects may be beneficial to the pathological process of ON. However, the excessive use of EPO can boost the hematocrit, which is detrimental for microcirculatory perfusion in ischemic situations, such as $\mathrm{ON}$, and which causes polycythemia, a condition with abnormally high levels of RBCs. Rezaeian et al (27) reported that the administration of 5 repeated doses of 500 units of EPO only marginally increased the hematocrit in C57BL/6 mice after a period of approximately 7 days, whereas the administration of 5,000 units 5 times significantly increased the hematocrit. It has been found that a significantly increased hematocrit can aggravate bone necrosis due to impaired rheology, i.e., decreased $\mathrm{RBC}$ velocity and nutritive perfusion and hyperviscosity, which is believed to be detrimental despite the anti-apoptotic and angiogenic effects mediated by EPO (27). Furthermore, a previous study demonstrated that, to prevent the detrimental effects of EPO, the treatment of anemia in chronic renal failure usually requires low doses of EPO between 350 and 400 units or less on a very repetitive base over several weeks or months (27). Therefore, we decided to administer 7 doses of (500 units/ $\mathrm{kg} /$ day) of EPO in our study. Blood analyses did not reveal a significantly higher RBC count in the EPO-treated animals compared with the untreated controls on weeks 0,2 and 4 (Fig. 3). The levels 

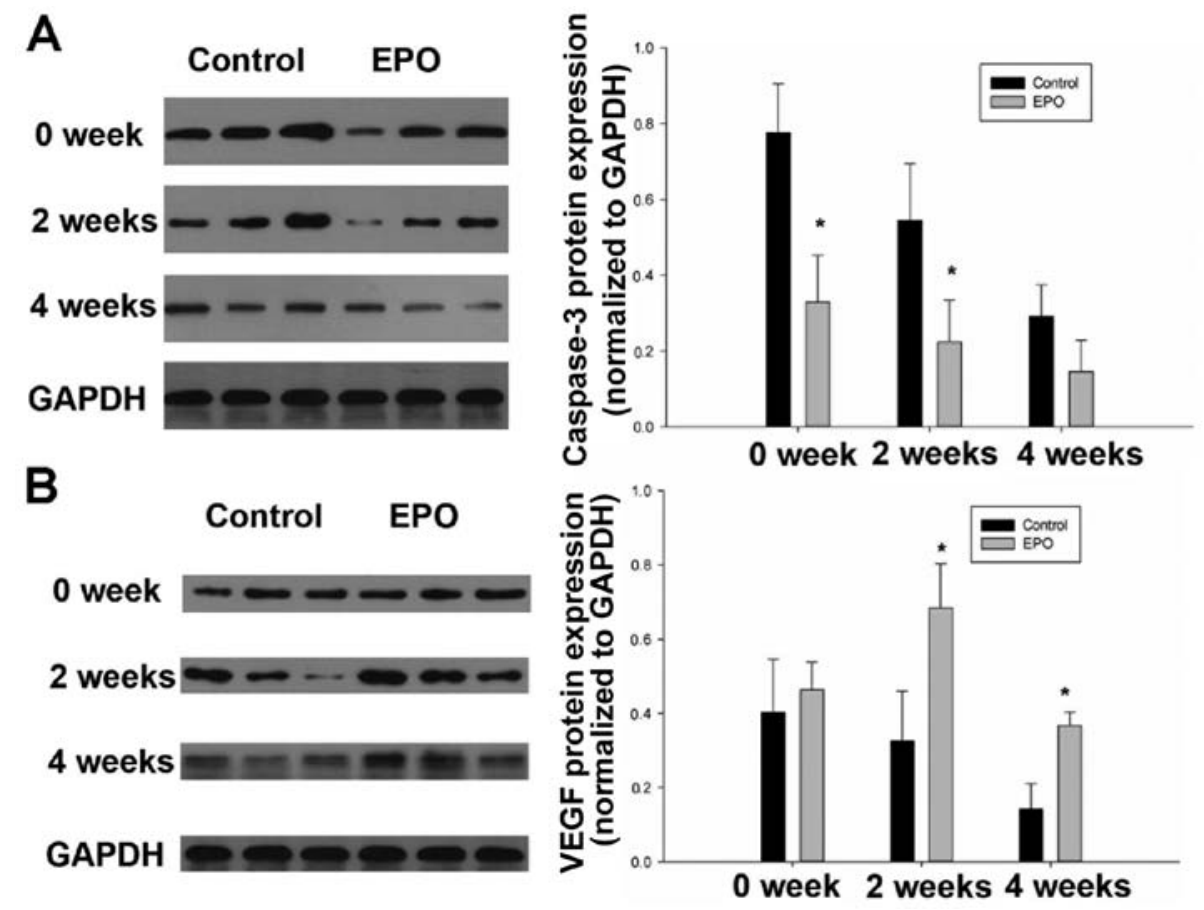

Figure 8. Immunoblot analyses of the expression of (A) caspase-3 and (B) VEGF in erythropoietin (EPO)-treated animals and the untreated controls at 0,2 and 10 weeks. Data are presented as the means $\pm \mathrm{SD}$. ${ }^{*} \mathrm{P}<0.05$ vs. controls.

of hemoglobin and hematocrit showed a similar trend (Fig. 3). Additionally, histological analysis revealed a lower ON index in the EPO group compared iwth the control group (Figs. 5 and 6). These results illustrate that the administration of EPO at the appropriate dosage and repetitive manner exerts beneficial effects rather than harmful effects on the pathological process of ON. In accordance with the former data, the apoptotic index of osteoblasts and osteocytes in the EPO group was greatly reduced compared with the controls (Fig. 7). Moreover, the expression of VEGF markeldy increased following treatment with EPO (Fig. 8B). In conclusion, the results from our study suggest that the tissue-protective function of EPO in GC-induced ON is possibly attributed to the combination of its anti-apoptotic and VEGF-enhancing effects.

GCs have been reported to elicit the apoptosis of osteoblasts and osteocytes through direct and indirect mechanisms. Osteoblasts and osteocytes can be the direct targets of GC action in vivo and excess levels of steroid hormones directly induce the apoptosis of these cell types (5). Alternatively, infarction, as well as oxygen and nutrient deprivation caused by a high-dose administration of GCs inevitably lead to the apoptosis of osteoblasts and osteocytes (7). The activation of caspase-3, a common downstream effector of apoptotic signaling pathways, including the involvement of direct and indirect mechanisms, is observed in the GC-induced apoptosis of osteoblasts and osteocytes $(38,39)$. Consistent with the data from other studies $(40,41)$, we also demonstrated that GC enhanced apoptosis, as well as caspase-3 expression in trabecular bone in a time-dependent manner (Figs. 7 and 8A). However, the elevated levels of apoptosis and caspase- 3 expression were significantly repressed in response to EPO administration (Figs. 7 and 8A). However, it remains to be determined whether caspase- 3 expression is causative to the apoptosis observed in the $\mathrm{ON}$ zone and whether caspase- 3 is a direct target of EPO. Our results demonstrate that the appropriate dose of EPO exerts anti-apoptotic effects on GC-induced ON.

A number of of studies have suggested that GC impairs angiogenesis and osteogenesis by suppressing the production of VEGF in the femoral head in GC-induced ON (13,42-46). It has been reported that GCs suppress angiogenesis in fetal metatarsals, as well as hypoxia-inducible factor-1 $\alpha$ transcription and VEGF production in osteoblasts and osteocytes (13). Osteoblasts derived from femoral heads have been shown to exhibit a decrease in VEGF expression within $24 \mathrm{~h}$ of incubation with GCs (43). Furthermore, it is likely that VEGF and its receptors play vital roles coupling osteogenic and angiogenic processes in adult bone during remodeling and repair processes (47). In an in vivo model of $\mathrm{ON}$, rabbits treated with depomedrol plus adrenocorticotropic hormone (ACTH) for 1 month displayed fewer signs of trabecular necrosis and an increased expression of VEGF in comparison to animals treated with the steroid, depomedrol, alone (48). In the present study, immunoblot analysis revealed an elevated VEGF expression in the ON zone in the group administered EPO (Fig. 8B). Moreover, a higher VEGF expression was usually accompanied with a better histological performance and a higher trabecular bone volume fraction of the femoral head (Figs. 5 and 6). Taken together, the data from our study suggest that the administration of EPO exerts angiogenic and osteogenic effects in ON through a VEGF-related mechanism.

In conclusion, the present study demonstrates that the administration of EPO exerts prominent protective effects against $\mathrm{GC}$-induced $\mathrm{ON}$ of the femoral head in rats by inhibiting the apoptosis of osteoblasts and osteocytes and increasing the expression of VEGF. Further investigations of the molecular 
basis of EPO-mediated anti-apoptosis and regeneration in $\mathrm{ON}$ are required in order to develop novel strategies for the prevention and treatment of GC-induced ON.

\section{Acknowledgements}

The authors thank Changgeng Xu, Lei Wang, Shuibing Wang and Xiaolin Wu (Renmin Hospital of Wuhan University, Wuhan, Hubei, P.R. China) for their assistance in the process of the present study. This study was supported by the Fundamental Research Funds for the Central Universities and Independent Research Project of Wuhan University for Graduate Students (no. 2012302020207) and the National Natural Science Foundation of China (no. 81301592).

\section{References}

1. Silvestrini G, Ballanti P, Patacchioli FR, et al: Evaluation of apoptosis and the glucocorticoid receptor in the cartilage growth plate and metaphyseal bone cells of rats after high-dose treatment with corticosterone. Bone 26: 33-42, 2000.

2. Weinstein RS, Nicholas RW and Manolagas SC: Apoptosis of osteocytes in glucocorticoid-induced osteonecrosis of the hip. J Clin Endocrinol Metab 85: 2907-2912, 2000.

3. Calder JD, Pearse MF and Revell PA: The extent of osteocyte death in the proximal femur of patients with osteonecrosis of the femoral head. J Bone Joint Surg Br 83: 419-422, 2001.

4. Zalavras C, Shah S, Birnbaum MJ and Frenkel B: Role of apoptosis in glucocorticoid-induced osteoporosis and osteonecrosis. Crit Rev Eukaryot Gene Expr 13: 221-235, 2003.

5. O'Brien CA, Jia D, Plotkin LI, et al: Glucocorticoids act directly on osteoblasts and osteocytes to induce their apoptosis and reduce bone formation and strength. Endocrinology 145: 1835-1841, 2004

6. Yun SI, Yoon HY, Jeong SY and Chung YS: Glucocorticoid induces apoptosis of osteoblast cells through the activation of glycogen synthase kinase 3beta. J Bone Miner Metab 27: $140-148,2009$

7. Kaushik AP, Das A and Cui Q: Osteonecrosis of the femoral head: an update in year 2012. World J Orthop 3: 49-57, 2012.

8. Moutsatsou P, Kassi E and Papavassiliou AG: Glucocorticoid receptor signaling in bone cells. Trends Mol Med 18: 348-359, 2012.

9. Kerachian MA, Seguin C and Harvey EJ: Glucocorticoids in osteonecrosis of the femoral head: a new understanding of the mechanisms of action. J Steroid Biochem Mol Biol 114: 121-128, 2009.

10. Weinstein RS: Glucocorticoid-induced osteonecrosis. Endocrine 41: 183-190, 2012.

11. Weinstein RS: Glucocorticoid-induced osteoporosis and osteonecrosis. Endocrinol Metab Clin North Am 41: 595-611, 2012.

12. Kerachian MA, Cournoyer D, Harvey EJ, et al: New insights into the pathogenesis of glucocorticoid-induced avascular necrosis: microarray analysis of gene expression in a rat model. Arthritis Res Ther 12: R124, 2010

13. Weinstein RS, Wan C, Liu Q, et al: Endogenous glucocorticoids decrease skeletal angiogenesis, vascularity, hydration, and strength in aged mice. Aging Cell 9: 147-161, 2010.

14. Gerber HP, Vu TH, Ryan AM, Kowalski J, Werb Z and Ferrara N: VEGF couples hypertrophic cartilage remodeling, ossification and angiogenesis during endochondral bone formation. Nat Med 5: 623-628, 1999.

15. Leist M, Ghezzi P, Grasso G, et al: Derivatives of erythropoietin that are tissue protective but not erythropoietic. Science 305: $239-242,2004$

16. Weng S, Zhu X, Jin Y, Wang T and Huang H: Protective effect of erythropoietin on myocardial infarction in rats by inhibition of caspase-12 expression. Exp Ther Med 2: 833-836, 2011.

17. Teixeira M, Rodrigues-Santos P, Garrido P, et al: Cardiac antiapoptotic and proproliferative effect of recombinant human erythropoietin in a moderate stage of chronic renal failure in the rat. J Pharm Bioallied Sci 4: 76-83, 2012.
18. Calvillo L, Latini R, Kajstura J, et al: Recombinant human erythropoietin protects the myocardium from ischemia-reperfusion injury and promotes beneficial remodeling. Proc Natl Acad Sci USA 100: 4802-4806, 2003.

19. Choi D, Schroer SA, Lu SY, et al: Erythropoietin protects against diabetes through direct effects on pancreatic beta cells. J Exp Med 207: 2831-2842, 2010.

20. Celik M, Gokmen N, Erbayraktar S, et al: Erythropoietin prevents motor neuron apoptosis and neurologic disability in experimental spinal cord ischemic injury. Proc Natl Acad Sci USA 99: 2258-2263, 2002.

21. Gorio A, Gokmen N, Erbayraktar S, et al: Recombinant human erythropoietin counteracts secondary injury and markedly enhances neurological recovery from experimental spinal cord trauma. Proc Natl Acad Sci USA 99: 9450-9455, 2002.

22. Xiong M, Chen S, Yu H, Liu Z, Zeng Y and Li F: Neuroprotection of erythropoietin and methylprednisolone against spinal cord ischemia-reperfusion injury. J Huazhong Univ Sci Technolog Med Sci 31: 652-656, 2011.

23. Imamura R, Moriyama T, Isaka Y, et al: Erythropoietin protects the kidneys against ischemia reperfusion injury by activating hypoxia inducible factor-1alpha. Transplantation 83: 1371-1379, 2007.

24. MacRedmond R, Singhera GK and Dorscheid DR: Erythropoietin inhibits respiratory epithelial cell apoptosis in a model of acute lung injury. Eur Respir J 33: 1403-1414, 2009.

25. Kakavas S, Demestiha T, Vasileiou $P$ and Xanthos $T$ : Erythropoetin as a novel agent with pleiotropic effects against acute lung injury. Eur J Clin Pharmacol 67: 1-9, 2011.

26. Galeano M, Altavilla D, Bitto A, et al: Recombinant human erythropoietin improves angiogenesis and wound healing in experimental burn wounds. Crit Care Med 34: 1139-1146, 2006.

27. Rezaeian F, Wettstein R, Amon M, et al: Erythropoietin protects critically perfused flap tissue. Ann Surg 248: 919-929, 2008.

28. Holstein JH, Orth M, Scheuer C, et al: Erythropoietin stimulates bone formation, cell proliferation, and angiogenesis in a femoral segmental defect model in mice. Bone 49: 1037-1045, 2011.

29. Yamamoto T, Irisa T, Sugioka Y and Sueishi K: Effects of pulse methylprednisolone on bone and marrow tissues: corticosteroidinduced osteonecrosis in rabbits. Arthritis Rheum 40: 2055-2064, 1997.

30. Qin L, Zhang G, Sheng H, et al: Multiple bioimaging modalities in evaluation of an experimental osteonecrosis induced by a combination of lipopolysaccharide and methylprednisolone. Bone 39: 863-871, 2006.

31. Ding S, Peng H, Fang HS, Zhou JL and Wang Z: Pulsed electromagnetic fields stimulation prevents steroid-induced osteonecrosis in rats. BMC Musculoskelet Disord 12: 215, 2011.

32. Sugano N, Kubo T, Takaoka K, et al: Diagnostic criteria for nontraumatic osteonecrosis of the femoral head. A multicentre study. J Bone Joint Surg Br 81: 590-595, 1999.

33. McGee-Lawrence ME, Stoll DM, Mantila ER, Fahrner BK, Carey HV and Donahue SW: Thirteen-lined ground squirrels (Ictidomys tridecemlineatus) show microstructural bone loss during hibernation but preserve bone macrostructural geometry and strength. J Exp Biol 214: 1240-1247, 2011.

34. Parfitt AM, Drezner MK, Glorieux FH, et al: Bone histomorphometry: standardization of nomenclature, symbols, and units. Report of the ASBMR Histomorphometry Nomenclature Committee. J Bone Miner Res 2: 595-610, 1987.

35. Yatsiv I, Grigoriadis N, Simeonidou C, et al: Erythropoietin is neuroprotective, improves functional recovery, and reduces neuronal apoptosis and inflammation in a rodent model of experimental closed head injury. FASEB J 19: 1701-1703, 2005.

36. Ribatti D, Presta M, Vacca A, et al: Human erythropoietin induces a pro-angiogenic phenotype in cultured endothelial cells and stimulates neovascularization in vivo. Blood 93: 2627-2636, 1999.

37. Erslev A: Humoral regulation of red cell production. Blood 8: 349-357, 1953.

38. Liu Y,Porta A,Peng X, et al: Prevention of glucocorticoid-induced apoptosis in osteocytes and osteoblasts by calbindin-D28k. J Bone Miner Res 19: 479-490, 2004.

39. Thornberry NA and Lazebnik Y: Caspases: enemies within. Science 281: 1312-1316, 1998.

40. Fukuzuka K, Edwards CK III, Clare-Salzler M, Copeland EM III, Moldawer LL and Mozingo DW: Glucocorticoid-induced, caspase-dependent organ apoptosis early after burn injury. Am J Physiol Regul Integr Comp Physiol 278: R1005-R1018, 2000. 
41. Gao YS, Guo SC, Ding $\mathrm{H}$ and Zhang CQ: Caspase-3 may be employed as an early predictor for fracture induced osteonecrosis of the femoral head in a canine model. Mol Med Rep 6: 611-614, 2012.

42. Wang G, Zhang CQ, Sun Y, et al: Changes in femoral head blood supply and vascular endothelial growth factor in rabbits with steroid-induced osteonecrosis. J Int Med Res 38: 1060-1069, 2010.

43. Varoga D, Drescher W, Pufe M, Groth G and Pufe T: Differential expression of vascular endothelial growth factor in glucocorticoid-related osteonecrosis of the femoral head. Clin Orthop Relat Res 467: 3273-3282, 2009.

44. Weinstein RS: Glucocorticoids, osteocytes, and skeletal fragility: the role of bone vascularity. Bone 46: 564-570, 2010.
45. Seamon J, Keller T, Saleh J and Cui Q: The pathogenesis of nontraumatic osteonecrosis. Arthritis 2012: 601763, 2012.

46. Wang Y, Wan C, Deng L, et al: The hypoxia-inducible factor alpha pathway couples angiogenesis to osteogenesis during skeletal development. J Clin Invest 117: 1616-1626, 2007.

47. Clarkin CE and Gerstenfeld LC: VEGF and bone cell signalling: an essential vessel for communication? Cell Biochem Funct 31: 1-11, 2013.

48. Zaidi M, Sun L, Robinson LJ, et al: ACTH protects against glucocorticoid-induced osteonecrosis of bone. Proc Natl Acad Sci USA 107: 8782-8787, 2010. 